\title{
Fourier transforms of fractional order and their optical interpretation
}

\author{
Haldun M. Ozaktas \\ Electrical Engineering, Bilkent University, 06533 Bilkent, Ankara, Turkey
}

and

David Mendlovic
Tel-Aviv University. Faculty of Engineering, 69978 Tel-Aviv, Israel

Received 11 February 1993; revised manuscript received 13 April 1993

\begin{abstract}
Fourier transforms of fractional order $a$ are defined in a manner such that the common Fourier transform is a special case with $\operatorname{order} a=1$. An optical interpretation is provided in terms of quadratic graded index media and discussed from both wave and ray viewpoints. Fractional Fourier transforms can extend the range of spatial filtering operations.
\end{abstract}

The original definition of the derivatives of a function makes sense only for integral orders, i.e. we can speak of the first or second derivative and so on. However, it is possible to extend the definition of the derivative to noninteger orders by using an elementary property of Fourier transforms. Bracewell shows how fractional derivatives can be used to characterize the discontinuitics of certain functions [1]. An example from the field of optics is related to the Talbot effect $[2]$, in which self-images of an input object are observed at the 2D planes $z=N z_{0}$ for integer $N\left(z\right.$ is the axial coordinate and $z_{0}$ a characteristic distance). Using a self-transformation technique [3], it was shown that $N$ could also take on certain rational values.

In this letter we define fractional Fourier transformations in a similar spirit. The ath Fourier transform of a function $f(x, y)$ will be denoted as $\mathscr{F}^{a}[f(x, y)]$, or simply $\mathscr{F}^{a} f$ when there is no room for confusion. We require that our definition satisfy two basic postulates. First, $\mathscr{F}^{\prime} f$ should be the usual first Fourier transform, defined as

$$
\begin{aligned}
& \left(\tilde{F}^{\prime} f\right)\left(x^{\prime}, y^{\prime}\right)=\int_{-\infty}^{+\infty} \int_{-\infty}^{+\infty} f(x, y) \\
& \times \exp \left[-\mathrm{i} 2 \pi\left(x^{\prime} x+y^{\prime} y\right) / s^{2}\right] \mathrm{d} x \mathrm{~d} y,
\end{aligned}
$$

where $s, x, x^{\prime}, y, y^{\prime}$ all have the dimensions of length. (The parentheses on the left hand side are to emphasize that the variables $x^{\prime}, y^{\prime}$ belong to $\mathscr{F}^{1} f$ and not $f$.) In a conventional " $2 f$ " optical Fourier transforming configuration ${ }^{\# 1}[4], x, y$ would denote the coordinate of the input plane, $x^{\prime}, y^{\prime}$ those of the Fourier plane, and $s^{2}=\lambda f$ ( $\lambda=$ wavelength of light, $f=$ focal length of lens).

Our second postulate is to require that

$$
\mathscr{F}^{a}\left[\mathscr{F}^{b} f\right]=\mathscr{F}^{a} \mathscr{F}^{b} f=\mathscr{F}^{b} \mathscr{F}^{a} f=\mathscr{F}^{a+b} f \text {. }
$$

Consistent with our two postulates, $\mathscr{F}^{1 / Q}[\cdot]$ may be defined for integer $Q$ as that operation which when applied $Q$ times gives the first (conventional) Fourier transform of $f$. An optical system which performs

\#1 By this we are referring to the common textbook configuration consisting of free-space propagation by a distance $f$, a lens of focal length $f$, and again free space propagation by a distance $f$. 
this operation may be realized by inserting a lens of appropriate focal length midway between the appropriately spaced input and output planes. It is now possible to define Fourier transforms of rational or$\operatorname{der} \mathscr{F}^{P / Q} f$ by repeated application of the operator $\mathscr{\mathscr { H }}^{1 / Q}$ for $P$ times. The definition can be generalized to real orders by a limiting process.

Here we will not pursue this line of thought. Instead, we recall that the optical Fourier transforming operation is a result of the joint action of the dual operations of free-space propagation and focusing [ 5,6$]$. In a conventional " $2 f$ " system, the focusing action is concentrated at the lens location. The same operation can be performed by $Q$ fractional Fourier transform stages in cascade each performing $\mathscr{F}^{1 / Q}[\cdot]$. In this latter system, the act of focusing is evenly distributed through the act of propagation. In the limit that $Q \rightarrow \infty$, focusing and propagation will be infinitesimally and uniformly interspersed between each other. Of course, bulk systems with even moderately large $Q$ would be quite impractical. Fortunately, systems satisfying this property can be realized as quadratic graded index (GRIN) media. Such media can be thought to consist of infinitesimal layers in which focusing and propagation take place simultaneously. The refractive index distribution in such a medium is given by [7]

$n^{2}(r)=n_{1}^{2}\left[1-\left(n_{2} / n_{1}\right) r^{2}\right]$,

where $r^{2}-x^{2}+y^{2}$ is the radial distance from the optical axis and $n_{1}, n_{2}$ are the GRIN medium parameters. By solving the ray equation, it was shown [7] that a parallel bundle of rays will be focused a distance $L \equiv(\pi / 2) \sqrt{n_{1} / n_{2}}$ away from the input plane. If a function $f(x, y)$ is presented at the input plane $z=0$, at the plane $z=L$ we observe $\mathscr{F}^{\mathrm{l}} f$ as given by eq. (1) [8]. (This confirms that $z=L$ is the focal plane not only from the ray optics point of view but also from the physical optics point of view.) Now, since the system is fully uniform in the axial direction, $\bar{F}^{a} f$ can be physically defined as the functional form of the scalar light distribution at $z=a L$.

Above, we have motivated and defined the fractional Fourier transform in physical terms. However, it is important to note that fractional Fourier transforms can be defined purely mathematically and GRIN media introduced afterwards as a physical interpretation. We now present such as mathemati- cal definition, also showing its relation to the physical definition above.

The self-modes of quadratic GRIN media are the 2D Hermite-gaussian ( $\mathrm{HG}$ ) functions [7], which form an orthogonal and complete basis set. The (l, $m$ )th member of this set is expressed as

$\Psi_{l m}(x, y)=H_{1}\left(\frac{\sqrt{2} x}{\omega}\right) H_{m}\left(\frac{\sqrt{2} y}{\omega}\right) \exp \left(-\frac{x^{2}+y^{2}}{\omega^{2}}\right)$

where $H_{l}$ and $H_{m}$ are Hermite polynomials of orders $l$ and $m$, respectively, $\omega=(2 / k)^{1 / 2}\left(n_{1} / n_{2}\right)^{1 / 4}$ with $k=2 \pi n_{1} / \lambda$, and $\lambda$ is the wavelength. Each HG mode propagates through the GRIN medium with a different propagation constant [7]

$$
\begin{aligned}
& \beta_{l m}=k\left[1-(2 / k)\left(n_{2} / n_{1}\right)^{1 / 2}(l+m+1)\right]^{1 / 2} \\
& \quad \approx k-\left(n_{2} / n_{1}\right)^{1 / 2}(l+m+1) .
\end{aligned}
$$

Any 2D function $f(x, y)$ can be expressed in terms of the HG basis set as

$$
\begin{aligned}
& f(x, y)=\sum_{l} \sum_{m} A_{l m} \Psi_{l m}(x, y), \\
& A_{l m}=\int_{-\infty}^{\infty} f(x, y)\left[\Psi_{l m}(x, y) / h_{l m}\right] \mathrm{d} x \mathrm{~d} y,
\end{aligned}
$$

where $h_{l m}=2^{(l+m)} l ! m ! \pi \omega^{2} / 2$.

Now, the fractional Fourier transform of $f(x, y)$ of order $a$ can be defined as

$$
\overline{\mathscr{H}}^{a}[f(x, y)]=\sum_{l} \sum_{m} A_{l m} \Psi_{l m}(x, y) \exp \left(\mathrm{i} \beta_{l m} a L\right) \text {. }
$$

It was shown [8] that our two postulates are satisfied by this definition, and that the scaling factor $s$ appearing in eq. (1) is given by $s=\omega \sqrt{\pi}$. In the same reference, we also discuss and prove some of the properties of fractional Fourier transforms, which are richer than those of the common Fourier transform $\$ 2$, and generalize to complex values of the order $a$ (which can he physically realized by attenuating media). We also define fractional convolutions (or correlations) through the equation

$$
\operatorname{CONV}^{a}[f, g]=\mathscr{F}^{-a}\left[\mathscr{F}^{a} f \times \mathscr{F}^{a} g\right] \text {. }
$$

\#2 For a list of properties of the common Fourier transform, see ref. [1]. 
In order to demonstrate the fractional Fourier transform operation, some computer simulations have been carried out for one-dimensional signals. Let us assume that the input function is the common rectangle function. Figure 1 shows this initial signal and the magnitude of its first order Fourier transform. The phase information of the first Fourier transform is shown in fig. 2. The coefficients $A_{l}$ for our specific example are shown in fig. 3 . This figure demonstrates the fact that in this example harmonics of higher orders $(>15)$ contain very little of the total energy. Figure 4 shows the fractional Fourier transform of order 0.25 . Note that this same distri- bution is also the fractional Fourier transform of orders $1.75,2.25, \ldots$. Figures 5 and 6 show the fractional Fourier transforms of our input function for the orders $0.5,1.5, \ldots$, and $0.75,1.25, \ldots$, respectively.

To gain some additional insight regarding fractional Fourier transforms and also show its relation to ray optics, we now provide a phase-space interpretation. Let a particular paraxial ray be characterized by its radial distance $r$ and slope $s$, both with respect to the optical axis at a particular axial position $z$. Then, the effect of passing through any optical system on this ray can be described by a movement in $r-s$ space. For instance, free-space prop-

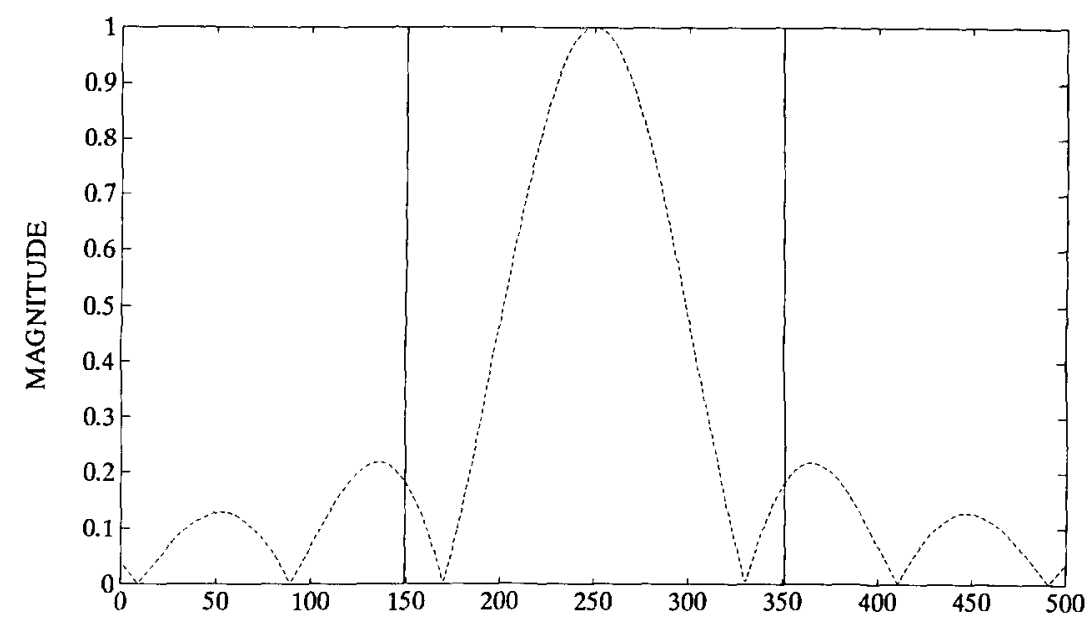

Fig. 1. Solid curve: input signal, dashed curve; magnitude of its first order Fourier transform.

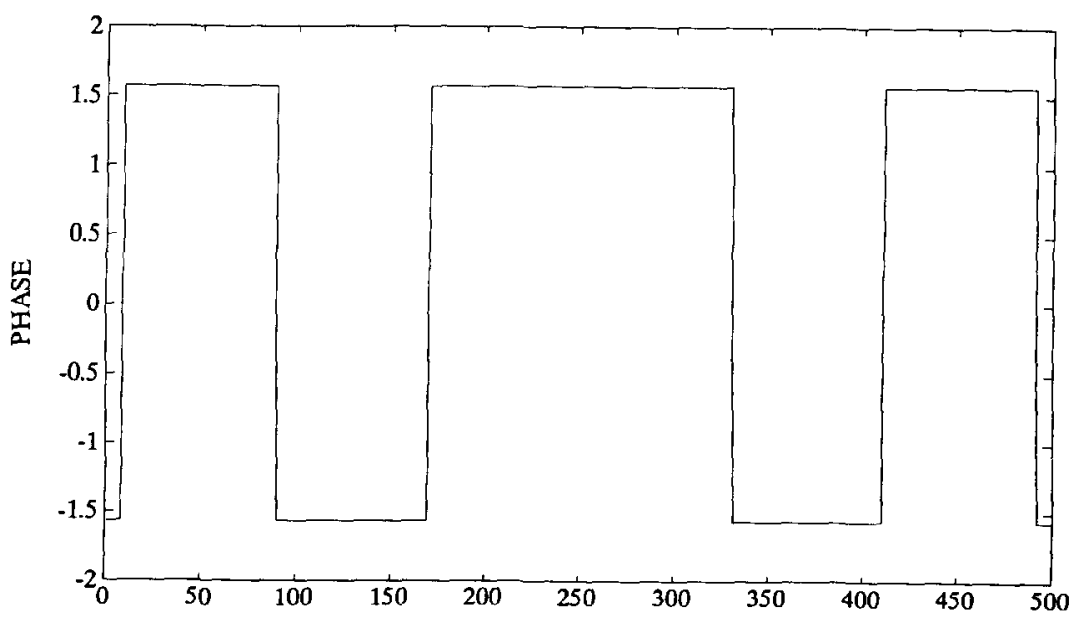

Fig. 2. Phase of the first order Fourier transform of the input signal in the previous figure. 


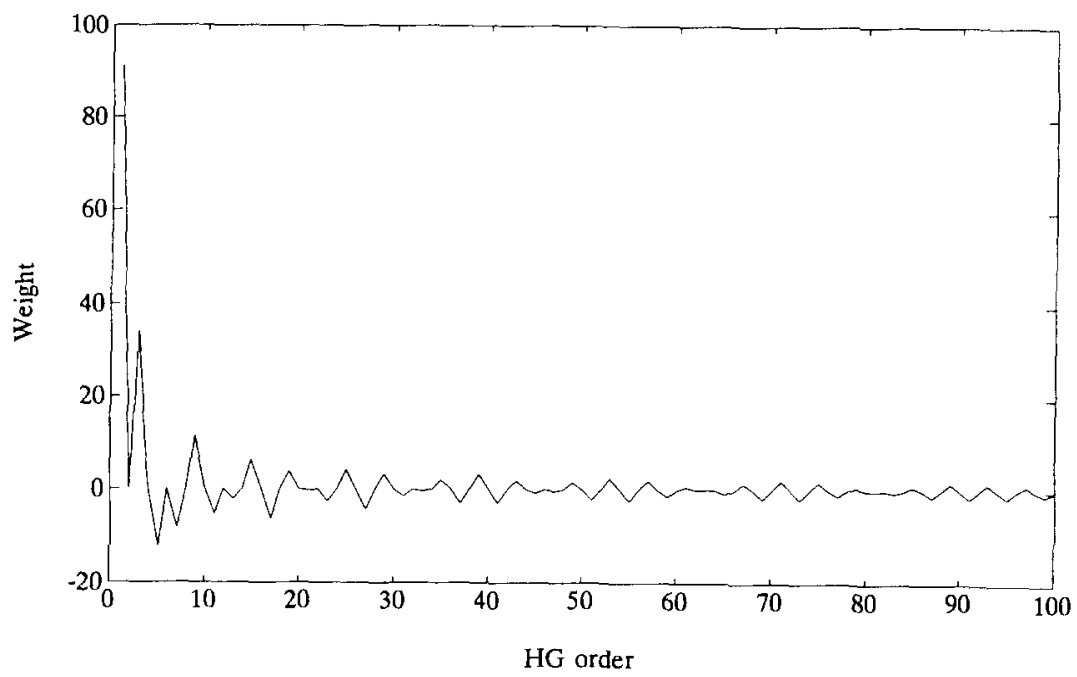

Fig. 3. Energy distribution among the first $100 \mathrm{HG}$ orders.
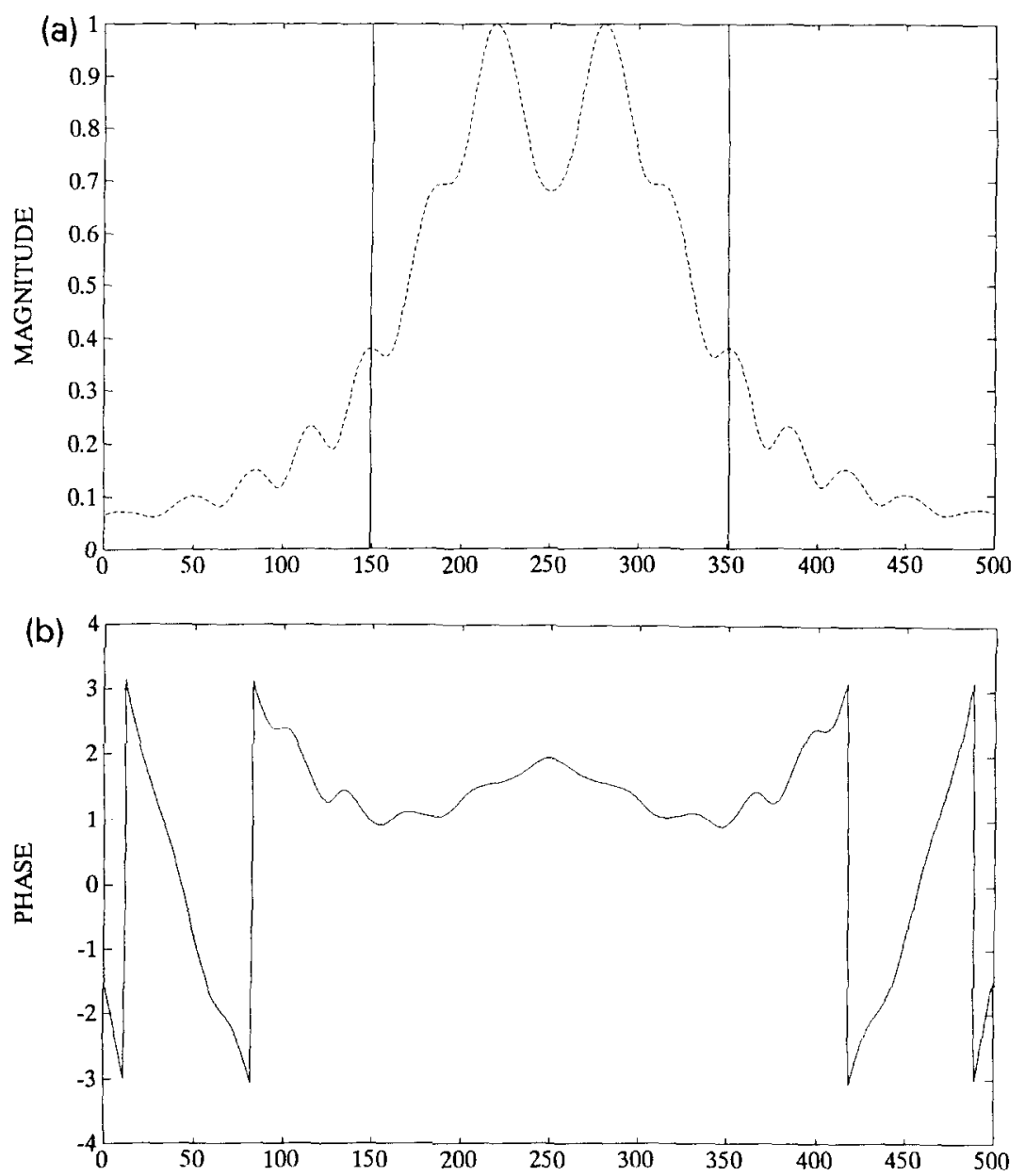

Fig. 4. Fractional Fourier transform of order $a=0.25$, (a) magnitude, (b) phase. 

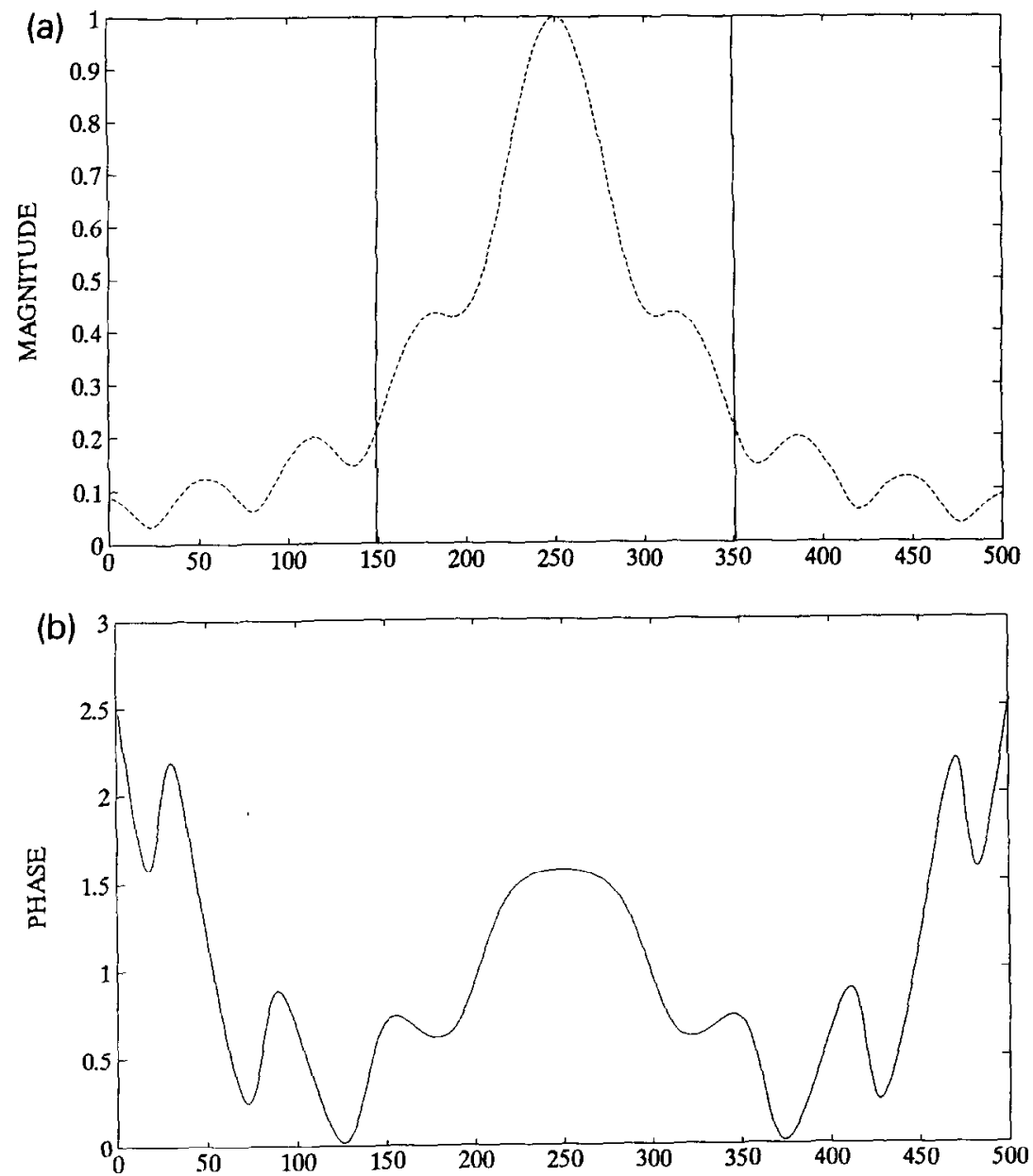

Fig. 5. Fractional Fourier transform of order $a=0.5$, (a) magnitude, (b) phase.

agation corresponds to a horizontal displacement whereas focusing by a lens corresponds to a vertical displacement. Let us now consider a bundle of rays with a uniform spread of $r$ and $s$ (represented by the shaded rectangular region in fig. 7a) and consider how this ray bundle is transformed as it passes through a conventional " $2 f$ " Fourier transforming configuration (fig. 7). The overall effect of the " $2 f$ " system is to rotate the rectangular region by $90^{\circ}$, although the intermediate steps result in shearing of the rectangular region.

How do things look like in phase space if we use quadratic GRIN media instead? It is known that $r$ and $s$ obey the following equations in such media [7]:
$r(z+\Delta z)=r(z) \cos (\pi \Delta z / 2 L)-s(z) \sin (\pi \Delta z / 2 L)$, $s(z+\Delta z)=r(z) \sin (\pi \Delta z / 2 L)+s(z) \cos (\pi \Delta z / 2 L)$,

from which we can conclude that the region representing any given bundle of rays in phase space is uniformly rotated as we go from $z=0$ to $z=L$. This uniform behavior is to be contrasted with that of the bulk " $2 f$ " Fourier transformer in which the focusing is concentrated at the lens, instead of being uniformly distributed throughout the system. This has discouraged us from basing the definition of fractional Fourier transforms on conventional bulk Fourier transformers. Although we cannot exclude 

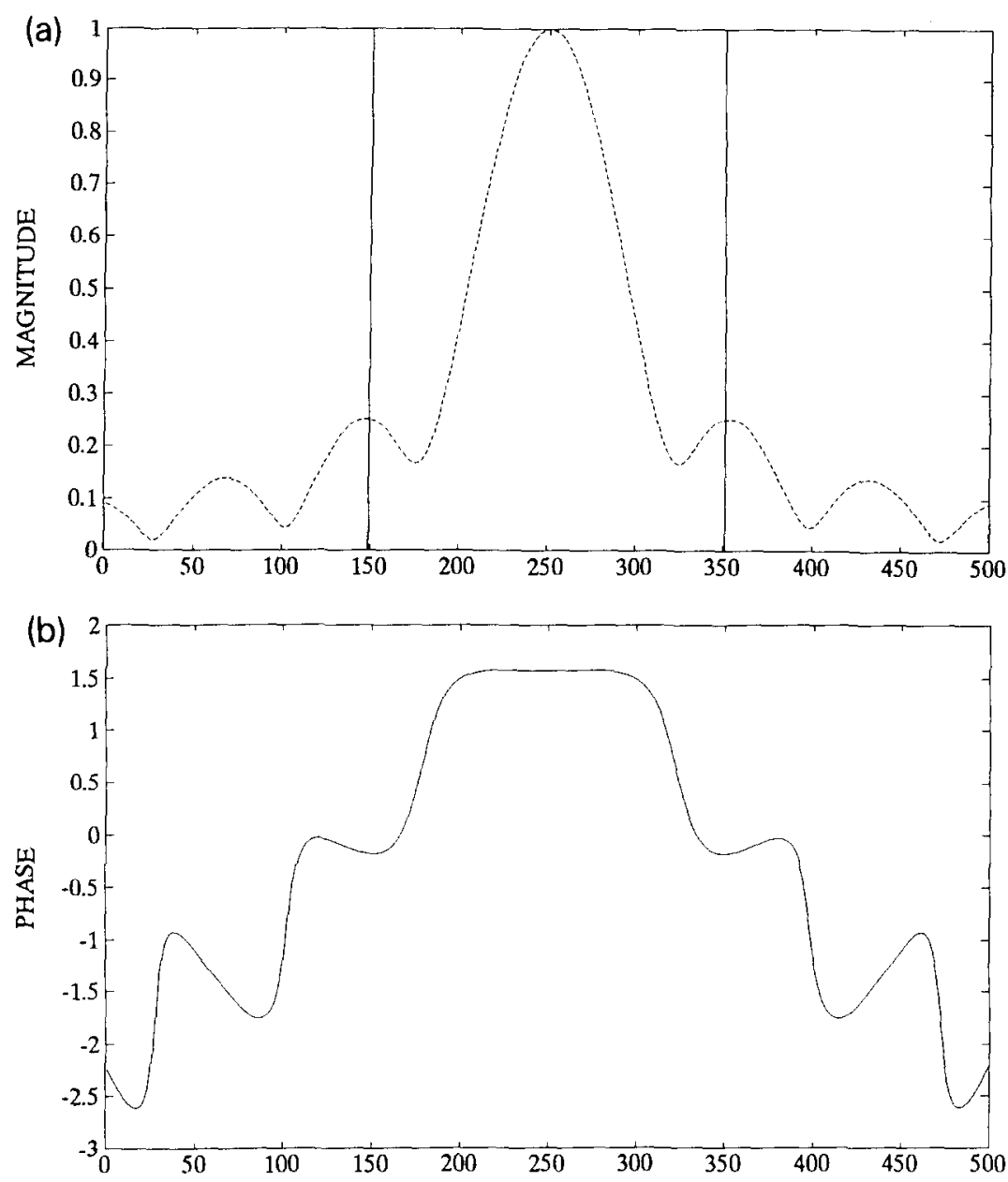

Fig. 6. Fractional Fourier transform of order $a=0.75$, (a) magnitude, (b) phase.

the possibility of other definitions consistent with our two postulates, our particular definition is seen to be a natural and meaningful one, especially in an optical context.

Fractional Fourier transforms can form the basis of generalized spatial filtering operations, extending the range of operations possible with optical information processing systems. Conventional Fourier plane filtering systems [4] are based on a spatial filter introduced at the Fourier plane. This limits the operations achievable to linear space-invariant ones (i.e. operations which can be expressed as a convolution of the input function with a space-invariant impulse response). By introducing several filters at different fractional Fourier planes, it may be possi- ble to implement a wider class of operation. Note that full space-variant operations can be implemented using approaches such as (or equivalent to) vectormatrix multiplier architectures [9] or multi-facet architectures [10], but these result in a heavy penalty in terms of space-bandwidth product utilization. A detailed treatment of how many filters are needed and how they should be synthesized to realize a given opcration must be postponed for future research. Here we must satisfy ourselves by noting that this process, in its extreme, constitutes a generalization of conventional planar spatial filtering to volume spatial filtering.

Despite the attractiveness of this concept, it is perhaps worth noting that there have been some related 


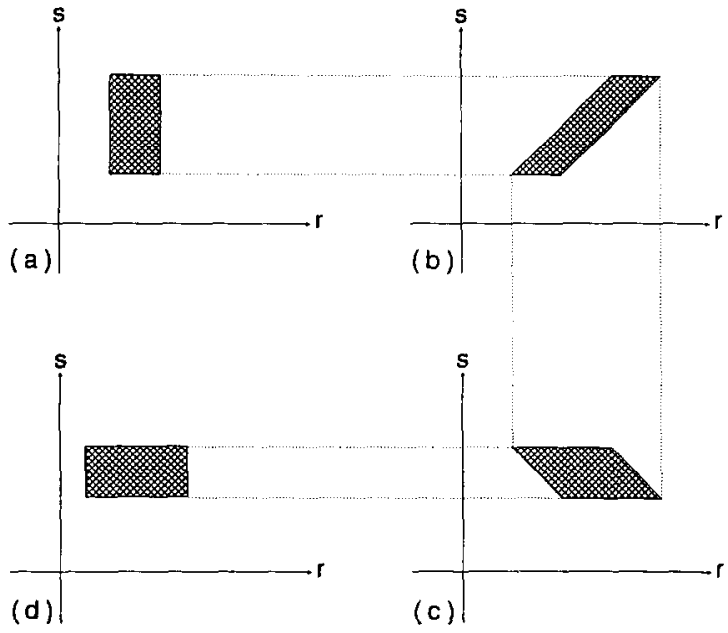

Fig. 7. (a) Phase space representation of the original bundle of rays. (b) After free space propagation through a distance $f$. (c) After passage through a lens of focal length $f$. (d) After another free space propagation through a distance $f$.

attempts (not based on the fractional Fourier transform in GRIN media as we have introduced it), which have been very difficult to implement with accurate results $[11,12]$. Thus, we refrain from optimistic conclusions regarding the usefulness of generalized spatial filtering before further results are developed.

A detailed mathematical treatment of the fractional Fourier transform will be given in another publication [8].
Some of the ideas in this letter were developed during intensive study sessions organized by Adolf W. Lohmann at the Applied Optics Group of the University of Erlangen-Nürnberg during the summer of 1992. David Mendlovic acknowledges a MINERVA fellowship and Haldun M. Ozaktas acknowledges an Alexander von Humboldt fellowship which made this cooperation possible.

\section{References}

[1] R. Bracewell, The Fourier transform and its applications, 2nd Ed. (McGraw-Hill, New York, 1986).

[2] K. Patorski, in: Progress in optics, Vol. 28, ed. E. Wolf (North-Holland, Amsterdam, 1989) p. 3.

[3] A. Lohmann and D. Mendlovic, Self-Fourier objects and other self-transform objects, J. Opt. Soc. Am. A 9 (1992).

[4] J. Goodman, Introduction to Fourier optics (McGraw-Hill, New York, 1968).

[5] A. Lohmann, Optik (Stuttgart) 11 (1954) 478 (in german); [Optik 89 (1992) 93].

[6] A. Vander Lugt, Optical signal processing (Wiley, New York, 199X).

[7] A. Yariv, Optical electronics, 3rd Ed. (Holt Sounders, 1985).

[8] D. Mendlovic and H.M. Ozaktas, Fractional Fourier transformations and their optical implementation: Part I, J. Opt. Soc. Am. A, accepted.

[9] J.W. Goodman, A.R. Dias and L.M. Woody, Optics Lett. 2 (1978) 1.

[10] S.K. Case, P.R. Haugen and O.J. Løberge, Appl. Optics 20 (1981) 2670.

[11] D. Gabor, IBM Journal of Research and Development 13 (1969) 156.

[12] Potaturkin and Tverdohleb, Avtometria 1 (1974) 31. 